\title{
MODELING He-RICH DISKS IN AM CVn BINARIES
}

\author{
T. Nagel, ${ }^{1}$ S. Dreizler, ${ }^{2}$ T. Rauch, ${ }^{3,1}$ and K. Werner ${ }^{1}$
}

We have developed a new code for the calculation of synthetic spectra and vertical structures of accretion disks in cataclysmic variables and compact X-ray binaries. Here we present results for the CV system AM CVn.

AM CVn stars are a special type of cataclysmic variables, also called helium cataclysmics. They are systems of interacting binary white dwarfs, consisting of a degenerate $\mathrm{C}-\mathrm{O}$ white dwarf primary and a low mass semi-degenerate secondary. The secondary loses mass, almost pure helium, to the primary, forming an accretion disk. They have all in common a helium-rich composition, analoguous to the hydrogen-rich cataclysmic variables. They show photometric variabilities on time scales of $\sim 1000 \mathrm{~s}$, the prototype of the class, AM CVn, e.g. exhibits a variability of $\sim 18 \mathrm{~min}$ (Nelemans et al. 2001).

In order to calculate the spectrum of the accretion disk of AM CVn, we assume a radial disk structure according to Shakura \& Sunyaev $(1973, \alpha$-disk models) and a stationary Keplerian motion. Then the dissipated and radiated energy depends only on the radial distance $R$ from the center of symmetry, as well as on mass and radius of the central object $\left(M_{\star}\right.$, $R_{\star}$ ), and the mass accretion rate $\dot{M}$. Assuming axial symmetry, we divide the disk into a set of concentric rings. For each ring, the equations of radiative and hydrostatic equilibrium as well as the rate equations are solved consistently with the radiation transfer equation under the constraint of particle number and charge conservation by an ALI technique (Werner \& Husfeld 1985). Metal-line blanketing and irradiation by the central object is taken into account. Finally, the spectra of the individual disk rings are integrated to a complete disk spectrum for different inclination angles, and the spectral lines are Doppler broadened according to the radial component of the Kepler rotation.

For the calculation, we assumed a central object of $M_{\star}=1.1 \mathrm{M}$. with a radius of $R_{\star}=4600 \mathrm{~km}$. The accretion rate is assumed to be $\dot{M}=3$.

\footnotetext{
${ }^{1}$ Institut für Astronomie und Astrophysik, Universität Tübingen, Sand 1, 72076 Tübingen, Germany (nagel@astro. uni-tuebingen.de).

${ }^{2}$ Universitätssternwarte Göttingen, Geismarlandstr. 11, 37083 Göttingen, Germany.

${ }^{3}$ Dr-Remeis-Sternwarte, Sternwartweg 7, 96049 Bamberg, Germany.
}

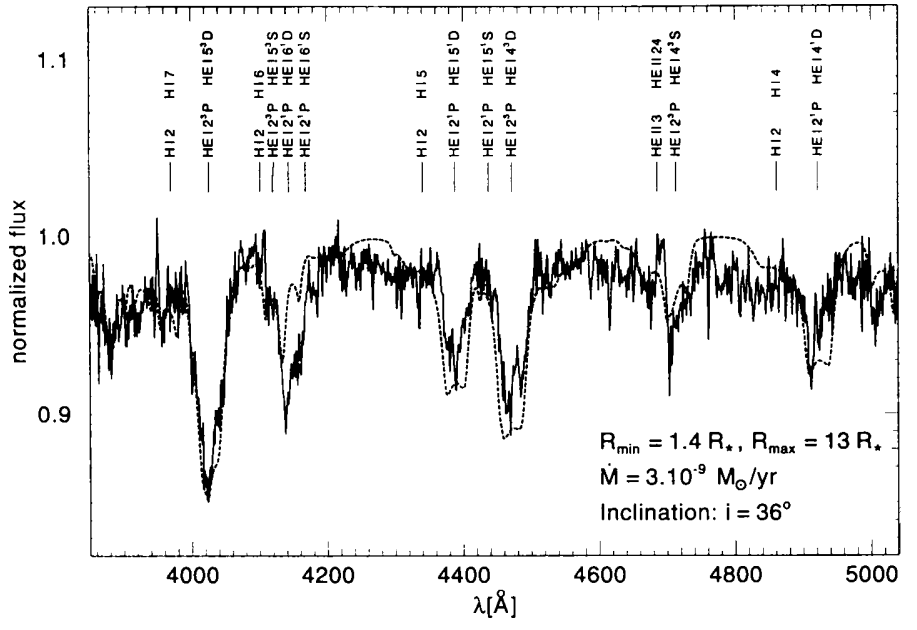

Fig. 1. Comparison of the normalized fluxes of an observed (solid line) and a synthetic spectrum (dashed line) of the AM CVn accretion disk. For the HeI $\lambda 4145 \AA$ line no detailed broadening tables have been available.

$10^{-9} \mathrm{M}_{\odot} / \mathrm{yr}$, following Nasser et al. (2001). The ratio of $\mathrm{H} / \mathrm{He}$ is $10^{-5}, \mathrm{C}-, \mathrm{N}$ - and $\mathrm{O}$-abundances are set to solar values. By comparing the calculated with an observed spectrum one can determine several parameters of the accretion disk, such as inner and outer radius, inclination angle, chemical composition or mass accretion rate.

We found the inner radius of the disk to be $1.4 R_{\star}$, an outer radius of $13 R_{\star}$, an inclination angle of $36^{\circ}$. Mass-accretion rate and chemical composition have not been varied during the calculations. Figure 1 displays a comparison of the normalized fluxes of an observed (solid) with a synthetic spectrum (dashed) of the AM CVn accretion disk.

\section{REFERENCES}

Nasser, M.R., Solheim, J.-E. \& Semionoff, D.A. 2001, A\&A, 373, 222

Nelemans, G., Portegies Zwart, S.F., Verbunt, F. et al. 2001, A\&A, 368, 939

Shakura, N.J., \& Sunyaev, R.A. 1973, A\&A, 24, 337

Werner, K. \& Husfeld, D. 1985, A\&A, 148, 417 